\title{
Accuracy of the serological detection of IgG and IgM to SARS-Cov-2: a prospective, cross-sectional study
}

\author{
Valentina Pecoraro ${ }^{1} \cdot$ Tiziana Cassetti $^{2} \cdot$ Marisa Meacci $^{3} \cdot$ Raffaele Gargiulo $^{2} \cdot$ Maria Rosaria Capobianchi $^{4}$. \\ Cristina Mussini ${ }^{5} \cdot$ Elena Vecchi $^{6} \cdot$ Monica Pecorari $^{7} \cdot$ Carlo Gagliotti $^{8} \cdot$ Tommaso Trenti $^{1} \cdot$ Mario Sarti $^{3}$
}

Received: 21 July 2020 / Accepted: 8 March 2021 / Published online: 10 April 2021

(C) The Author(s), under exclusive licence to Springer-Verlag GmbH Germany, part of Springer Nature 2021

\begin{abstract}
In response to the rapidly evolving of SARS-CoV-2 infection, numerous serological tests have been developed but their sensitivity and specificity are unclear. We collected serum samples of patients and health-care professionals to assess the accuracy of chemiluminescent (CLIA) and two lateral flow immunochromatographic assays (LFIA) to determine IgG and IgM antibodies to SARS-CoV-2 virus. We calculated the $\varphi$ correlation for qualitative results and test accuracy, adopting the following case definition: either real-time-PCR positivity or serological positivity with at least two different tests. We analyzed 259 samples, obtaining strong correlation between CLIA and both LFIA for $\operatorname{IgG}(\varphi=0.9)$, and moderate correlation for $\operatorname{IgM}(\varphi=0.6)$. For patients, the sensitivity was suboptimal for all methods (CLIA $81 \%$, LFIA A 85\%, LFIA B 78\%), while it was poor in asymptomatic health-care workers (CLIA 50\%, LFIA A 50\%, LFIA B 33\%). Overall, CLIA is more sensitive and specific for the determination of both IgG and IgM, whilst both LFIA methods reported good sensitivity and specificity for IgG, but scarce sensitivity for the IgM determination. The determination of SARS-CoV-2-specific IgG is useful to detect infection 6 days from symptom onset.
\end{abstract}

Keywords SARS-CoV-2 $\cdot$ COVID-19 $\cdot$ Accuracy $\cdot \operatorname{IgG}$ to SARS-CoV-2 $\cdot \operatorname{IgM}$ to SARS-CoV-2

Valentina Pecoraro

v.pecoraro@ausl.mo.it

Tiziana Cassetti

tizianacassettibio@gmail.com

Marisa Meacci

meacci.marisa@aou.mo.it

Raffaele Gargiulo

r.gargiulo@ausl.mo.it

Maria Rosaria Capobianchi

maria.capobianchi@inmi.it

Cristina Mussini

cristina.mussini@unimore.it

Elena Vecchi

vecchi.elena@policlinico.mo.it

Monica Pecorari

pecorari.monica@policlinico.mo.it

Carlo Gagliotti

carlo.gagliotti@regione.emilia-romagna.it
Tommaso Trenti

t.trenti@ausl.mo.it

Mario Sarti

sarti.mario@aou.mo.it

1 Department of Laboratory Medicine and Pathology, Azienda USL of Modena, Modena, Italy

2 Clinical Microbiology Unit, Azienda USL Modena, Modena, Italy

3 Clinical Microbiology Unit, University Hospital Polyclinic, Modena, Italy

4 National Institute for Infectious Diseases "L. Spallanzani”, Rome, Italy

5 Infectious Diseases Clinic, University Hospital Polyclinic, Modena, Italy

6 Hospital Hygiene, University Hospital Polyclinic, Modena, Italy

7 Virology and Molecular Microbiology Unit, University Hospital Polyclinic, Modena, Italy

8 Regional Agency for Health and Social Care of Emilia-Romagna, Bologna, Italy 


\section{Introduction}

The 2019 novel coronavirus (SARS-CoV-2), causing the coronavirus disease-2019 (COVID-19), has created an explosive and global status of emergency. At present, there are still many knowledge gaps not only about the pathophysiology of different clinical severity but also about the immune status of patients affected by SARS-CoV-2.

The etiological agent's viral genome is around $30 \mathrm{~kb}$, which encodes for both structural and non-structural proteins. The structural proteins, such as membrane (M), envelope (E) protein, nucleocapsid $(\mathrm{N})$ protein, and spike protein $(\mathrm{S})$, are essential for maintaining both virus morphology and stability and to mediate attachment and entry into the host cells. Infection begins with the interaction of the receptor binding domain located in the $\mathrm{S}$ protein and the target receptor on the host cell surface, that is the angiotensin converting enzyme 2 (ACE2) for SARS-CoV-2 [1]. Viral infection can stimulate and activate the immune system cells into producing specific antibodies (Abs). In particular, Abs directed against the spike protein (S) of SARS-CoV-2 have been identified [2]. Serological tests searching for specific Abs are available for clinical laboratories. However, given that SARS-CoV-2 is a newly emerging virus, the dynamic of antibody response during the clinical course of COVID-19 is still unclear [3].

In this study, we aim to establish serum IgG and IgM detection performance of a conventional automated chemiluminescent immunoassay (CLIA) and two lateral flow immunochromatographic assays (LFIA) to diagnose SARSCoV-2 infection in hospitalized patients with suspected SARS-Cov-2 infection and health care workers.

\section{Methods}

The study was conducted in accordance with the International Coordinating Council for Clinical Trials and the Helsinki Declaration and was approved by the Local Ethics Committee (No. 396/2020/OSS/AOUMO). Reporting was compliant with STARD guidelines [4].

\section{Patients and sample collection}

From March 1 to 31, 2020, we collected samples from patients admitted to infectious disease clinics or intensive care unit with symptoms of COVID-19 (defined according to the European Centre for Disease Prevention and Control criteria [5]) and asymptomatic healthcare workers, including physicians and nurses employed in the provincial hospitals involved in a SARS-CoV-2 screening procedure. Additionally, sera from 50 patients admitted to hospital, prior to the SARSCoV-2 transmission in Italy (collected between October and December 2019), were included as negative controls. We collected demographic (age, gender), clinical (date of symptom onset), and testing (date of oropharyngeal and nasopharyngeal swab and serological testing) data.

In order to diagnose the SARS-CoV-2 infection on the day of admission (day 0), samples of nasopharyngeal and/or oropharyngeal swabs for real time (RT-PCR) and serum or plasma samples were obtained from hospitalized patients and asymptomatic health care workers during the study period. Furthermore, serum or plasma samples were collected every $2-4$ days for 8 hospitalized patients.

\section{Real Time PCR for detection of SARS-CoV-2 RNA}

Total RNA was extracted from the clinical samples using a commercial RNA-extraction kit and was reverse transcribed; then, the cDNA was amplified by real-time qualitative RTPCR, using a commercial kit in accordance with the manufacturer's instructions (Alinity m SARS-CoV-2 Assay-Abbott Molecular).

\section{Detection of IgG and IgM antibodies to SARS CoV-2}

Detection of specific IgG and IgM antibodies to SARS-CoV-2 was performed for all serum or plasma samples (patients, health care workers, and negative controls) with chemiluminescent assay (CLIA) and two different lateral flow immunochromatographic assays (LFIA). The procedures and the interpretation of the results were performed according to manufacturers' instructions.

Chemiluminescent immunoassay (CLIA): we used an automated assay (iFlash 1800-YHLO Biotech Co., Ltd., Shenzhen, China). Patient samples were incubated with SARS-CoV-2 antigen-coated paramagnetic microparticles that react to form antigen-antibody complex. Under magnetic field, magnetic particles are absorbed to the inner wall of reaction tubes and unbound material is washed away from the solid phase in a magnetic field. Then, acridinium-labeled anti-human $\operatorname{IgG}$ or IgM conjugates are added to form an antigen-antibody anti-human IgG and IgM antibody complex. After a second wash, the results of chemiluminescent reaction are measured $30 \mathrm{~min}$ after sample loading as relative light units (RLUs). A direct relationship exists between the amount of SARS-CoV-2 IgG and IgM Abs in the sample and the RLUs detected. The concentration of SARS-CoV-2 IgM or IgG Abs (AU/ml) was automatically calculated according to RLU and a built-in calibration curve, and $10.0 \mathrm{AU} / \mathrm{ml}$ was considered the positive cut-off; reactive result $(\geq 10.0 \mathrm{AU} /$ $\mathrm{mL}$ ) indicates potential infection by SARS-CoV-2 virus, and non-reactive results $(<10.0 \mathrm{AU} / \mathrm{mL})$ indicate no presence of SARS-CoV-2 infection.

Lateral flow immunochromatographic assays (LFIA): We used two different rapid tests for the qualitative detection of IgG and IgM against SARS CoV-2 in serum or plasma 
specimens: LFIA kit A (COVID-19 IgG/IgM Rapid Test Prima Lab SA) and LFIA kit B (Techno Genetics KHB Group-Shangai). LFIA tests do not require sophisticated laboratory equipment, and specific $\operatorname{IgG}$ and $\operatorname{IgM}$ are visually detected by the appearance of a colored band after a short incubation period of about $15 \mathrm{~min}$. Ten microliter of serum or plasma sample was inserted into the sample port, and 2 or 3 drops $(70-100 \mu \mathrm{l})$ of dilution buffer were added. Results are interpreted according to the appearance of colored lines.

\section{Statistical analysis}

Correlation between dichotomous variables (positive or negative result) was assessed with the $\varphi$ test, $95 \%$ confidence interval (CI). $\varphi<0$ suggests negative association, $\varphi>0$ suggests positive association, and $\varphi=0$ suggests lack of association. The correlation was defined as strong $(\varphi \geq 0.7)$, moderate $(0.0 .69 \geq \varphi \geq 0.4)$, or weak $(\varphi<0.4)$. Categories of time from symptom onset were based on days: 0-6 (category 1), 7-13 (category 2), 14-20 (category 3 ), and $\geq 21$ (category 4).

For all patients, data from serological tests were compared to molecular results in order to create a $2 \times 2$ table and to calculate sensitivity, specificity, positive predictive value (PPV), and negative predictive value (NPV) of IgG and IgM detected with CLIA, LFIA kit A, and LFIA kit B. For health care workers, the $2 \times 2$ table was created considering the subset of subjects who performed serological test \pm 3 days from molecular tests. A positive to SARS-Cov-2 infection was considered either a positive molecular test or two different positive serological tests. The association of ordinal variable to the outcome was assessed using a chi square test for trend. $P<0.05$ was considered to be statistically significant. Analyses were performed using the software MedCalc (v 19.2.1).

\section{Results}

\section{Characteristics of participants}

Overall, 259 subjects were included in this study, comprising 146 patients with symptoms of COVID-19 and 113 asymptomatic health care workers, and were tested with RT-PCR swab analysis and serological test. Subjects' demographic, clinical, and molecular test results are described in Table 1. All 50 negative control serum samples resulted negative to all serological tests. RT-PCR detection of SARS-CoV-2 RNA identified 75 participants (28.9\%; 70 patients and 5 health care workers) with positive swab results.

\section{Detection of IgG and IgM antibodies to SARS-CoV-2}

Overall subjects: According to CLIA analysis, participants with negative results had median $\operatorname{IgG}$ and IgM values of 0.66 AU/ml (range 0.12-7.7 AU/ml), and $0.83 \mathrm{AU} / \mathrm{ml}$ (range $0.2-8.51 \mathrm{AU} / \mathrm{ml}$ ), respectively. In participants with positive results, the median $\operatorname{IgG}$ and $\operatorname{IgM}$ values were $55.4 \mathrm{AU} / \mathrm{ml}$ (range from 14.5 to $132.9 \mathrm{AU} / \mathrm{ml}$ ), and $36.8 \mathrm{AU} / \mathrm{ml}$ (range 13.4-971.77 AU/ml), respectively.

The number of subjects with detectable specific Abs varied among CLIA, LFIA kit A, and LFIA kit B tests. Overall subjects with IgG and IgM positivity were recorded for 57 (22\%), $40(15.4 \%)$, and $28(10.8 \%)$, accordingly. IgG positive and IgM negative were $17(6.5 \%), 42(16.2 \%)$, and $43(16 \%)$ respectively, whereas IgG negative and IgM positive were 4 $(1.5 \%), 10(3.8 \%)$, and $5(1.9 \%)$, respectively (Table 2; Fig. $1)$.

Among the 75 subjects with positive swabs results, we observed IgG positive and IgM negative results in $13(17 \%)$, $28(37 \%)$, and $33(44 \%)$, respectively. Furthermore, we observed IgG negative and IgM positive in $2(2.5 \%), 3(4 \%)$, and $0(0 \%)$ subjects, respectively (Table 2$)$.

The correlation between CLIA and both LFIA kits was strong for $\operatorname{IgG}(\varphi=0.9$ and 0.89 , respectively), and moderate for $\operatorname{IgM}(\varphi=0.6$ and 0.55 , respectively). Likewise, the correlation between LFIA kit A and kit B was strong for IgG and moderate for IgM (Table 3 ).

Patients with symptoms of COVID-19: Among 146 patients, positive serological tests were documented for 73 (50\%), 82 (72\%), and $73(50 \%)$, respectively (Table 2). In particular, IgG positive and IgM negative were detected in 15 (10\%), 37 (25\%), and 41 (28\%), respectively (Table 2). An RT-PCR-positive swab was registered for 70 patients, and of these $55(78.5 \%), 58(83 \%)$ and $51(73 \%)$ were also found positive at the respective serological tests (Table 2).
Table 1 Overall and subgroup demographic and clinical characteristics and molecular test results

\begin{tabular}{llll}
\hline & Overall & Patients & Health care workers \\
\hline Total, $n$ & 259 & 146 & 113 \\
Gender, M/F & $115 / 144$ & $86 / 60$ & $29 / 84$ \\
Age, median (range) & $58(22-97)$ & $71(22-97)$ & $41(23-63)$ \\
Symptomatic subjects, $n(\%)$ & $146(56)$ & $146(100)$ & $0(0)$ \\
RT-PCR positive swab, $n(\%)$ & $75(29)$ & $70(48)$ & $5(4)$ \\
\hline
\end{tabular}

Abbreviations: RT-PCR real-time PCR 
Table 2 Overall IgG and IgM antibody detection to SARS-Cov-2 with CLIA and LFIA assays, and for subjects with RT-PCR positive swabs

\begin{tabular}{|c|c|c|c|c|c|c|c|c|c|}
\hline & CLIA & LFIA kit A & LFIA kit B & CLIA & LFIA kitA & LFIA kitB & CLIA & LFIA kit A & LFIA kit B \\
\hline \multicolumn{10}{|l|}{ All subjects } \\
\hline & \multicolumn{2}{|c|}{ Overall $(n=259)$} & & \multicolumn{3}{|c|}{ Patients $(n=146)$} & \multicolumn{3}{|c|}{ Health care workers $(n=113)$} \\
\hline $\mathrm{IgG}+/ \operatorname{IgM}+$ & 57 & 40 & 28 & 54 & 36 & 27 & 3 & 4 & 1 \\
\hline IgG+/IgM- & 17 & 42 & 43 & 15 & 37 & 41 & 2 & 5 & 2 \\
\hline IgG-/IgM+ & 4 & 10 & 5 & 4 & 9 & 5 & 0 & 1 & 0 \\
\hline IgG-/IgM- & 181 & 167 & 183 & 73 & 64 & 73 & 108 & 103 & 110 \\
\hline \multicolumn{10}{|c|}{ Subjects with RT-PCR-positive swabs } \\
\hline & \multicolumn{2}{|c|}{ Overall $(n=75)$} & & \multicolumn{3}{|c|}{ Patients $(n=70)$} & \multicolumn{3}{|c|}{ Health care workers $(n=5)$} \\
\hline $\mathrm{IgG}+/ \operatorname{IgM}+$ & 42 & 29 & 19 & 41 & 28 & 19 & 1 & 1 & 0 \\
\hline $\mathrm{IgG}+/ \operatorname{IgM}-$ & 13 & 28 & 33 & 12 & 27 & 32 & 1 & 1 & 1 \\
\hline IgG-/IgM+ & 2 & 3 & 0 & 2 & 3 & 0 & 0 & 0 & 0 \\
\hline IgG-/IgM- & 18 & 15 & 23 & 15 & 12 & 19 & 3 & 3 & 4 \\
\hline
\end{tabular}

Abbreviations: CLIA chemiluminescent assay, LFIA lateral flow immunochromatographic assay, RT-PCR real-time PCR

According to time from symptom onset, less than one-third of patients positive at RT-PCR were identified with IgG or IgM positive within 6 days with any serological test. Between 7 and 13 days, $77 \%$ of patients were positive for IgG with all tests. After 14 days, $\operatorname{IgG}$ positive was registered for $87-92 \%$, and IgM positive ranged from 42 to $83 \%$ (Table 4 ). The percentage of patients with 2 serological positive tests increased from 42 to $100 \%$ as time increased. However, about one-third of patients negative at RT-PCR were found positive for IgG or IgM with serological tests, without differences registered according to time (Table 4). Patients with $\geq 2$ positive serological tests ranged from 19 to $35 \%$ within 21 days and 2 of the 3 patients with testing after 21 days had 2 positive serological results.

The correlation between CLIA and both LFIA kits was strong for $\operatorname{IgG}$ ( $\varphi=0.92$ and 0.87 , respectively), and moderate for IgM; the correlation between LFIA kit A and kit B was strong for IgG and moderate for IgM (Table 3).

Among the 8 patients with multiple serological tests, 4 patients with negative tests at day 0 , registered a positive serological result on day 7 .

Health-care professionals: We included 113 asymptomatic health care workers (physicians and nurses), and $5(4 \%)$ were confirmed positive with RT-PCR swab analysis. Overall, the majority were negative at serological testing; $95 \%, 91 \%$ and 97\%, respectively (Table 2). Of the 5 patients confirmed positive with RT-PCR swab, 3 were negative for IgG and IgM with CLIA and LFIA kit A, and 4 with LFIA kit B.

The correlation between CLIA and both LFIA tests was strong for $\operatorname{IgG}$ and weak or moderate for IgM, whereas the correlation between LFIA kit A and kit B was moderate for both IgG and IgM (Table 3).
Fig. 1 Detection of anti-SARSCoV-2 IgG and IgM results

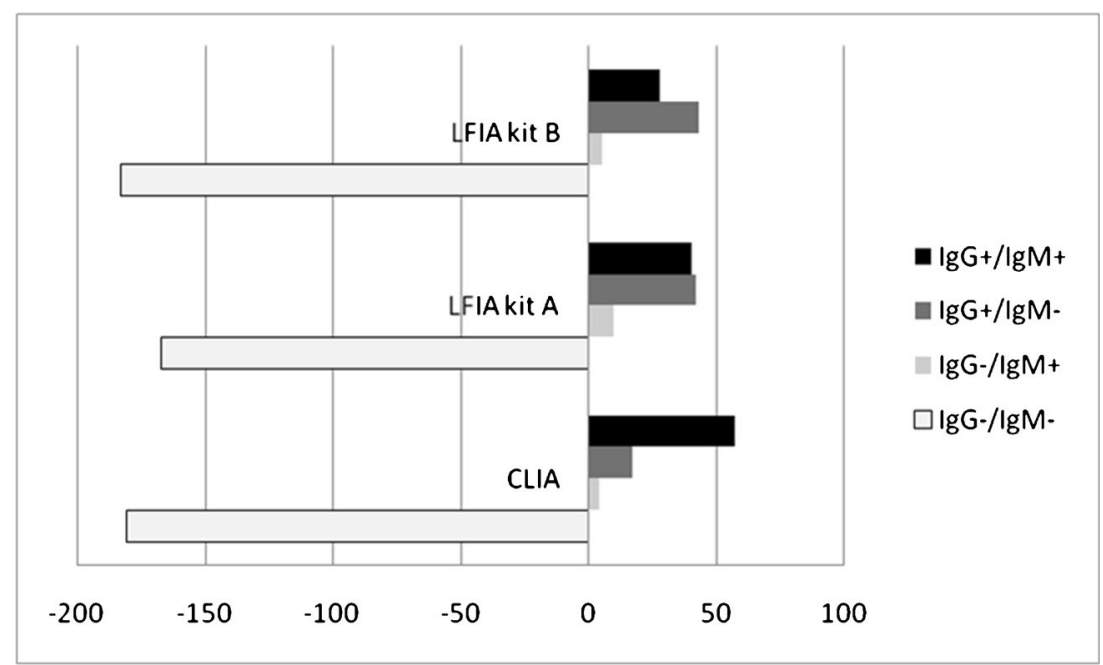


Table 3 Correlation analysis

\begin{tabular}{|c|c|c|c|c|c|c|}
\hline & \multicolumn{2}{|l|}{ Overall $(n=259)$} & \multicolumn{2}{|l|}{ Patients $(n=146)$} & \multicolumn{2}{|c|}{ Health care workers $(n=113)$} \\
\hline & $\operatorname{IgG}$ & $\operatorname{IgM}$ & $\operatorname{IgG}$ & $\operatorname{IgM}$ & $\operatorname{IgG}$ & $\operatorname{IgM}$ \\
\hline & $\varphi(95 \% \mathrm{CI})$ & $\varphi(95 \% \mathrm{CI})$ & $\varphi(95 \% \mathrm{CI})$ & $\varphi(95 \% \mathrm{CI})$ & $\varphi(95 \% \mathrm{CI})$ & $\varphi(95 \% \mathrm{CI})$ \\
\hline CLIA vs LFIA kit A & $0.90(0.87-0.92)$ & $0.60(0.49-0.65)$ & $0.92(0.89-0.94)$ & $0.55(0.42-0.65)$ & $0.73(0.63-0.80)$ & $0.23(0.05-0.40)^{*}$ \\
\hline CLIA vs LFIA kit B & $0.89(0.86-0.91)$ & $0.55(0.46-0.63)$ & $0.87(0.83-0.90)$ & $0.48(0.34-0.6)$ & $0.76(0.67-0.83)$ & $0.57(0.43-0.68)$ \\
\hline LFIA kit A vs LFIA kit B & $0.86(0.82-0.89)$ & $0.66(0.6-0.7)$ & $0.88(0.83-0.91)$ & $0.650 .54-0.73)$ & $0.56(0.42-0.67)$ & $0.44(0.28-0.58)$ \\
\hline
\end{tabular}

Abbreviations: CLIA chemiluminescent, LFIA lateral flow immunochromatographic assay

$* p=0.0143$

\section{Accuracy of Abs IgG and IgM to SARS-CoV-2}

For all subjects (patients, healthcare workers, and negative controls), the highest sensitivity was registered for LFIA kit A $(83.2 \%)$, followed by CLIA $(78.9 \%)$ and LFIA kit B (75.8\%). Specificity was highest for CLIA and LFIA kit B (99\% and 98\% respectively) followed by LFIA kit A (95\%) (Table 5). PPV were highest for CLIA (>98\%).

Among the symptomatic population, the highest sensitivity was registered for LFIA kit A (85.4\%), followed by CLIA $(81 \%)$ and LFIA kit B (78.6\%). Specificity was highest for CLIA and LFIA kit B (98\% and 94\% respectively) followed by LFIA kit A (89\%). PPV were highest for CLIA (both 98\%) (Table 5).

However, according to time from symptom onset, serological tests showed an excellent sensitivity and specificity when performed after 14 days from onset of symptoms, ranging from 93.7 to $100 \%$ (Supplemental Table).

For the 75 asymptomatic healthcare workers performing serological test \pm 3 days from the RT-PCR, the highest sensitivity was registered for CLIA and LFIA kit A (50\%), with the lowest sensitivity registered by LFIA kit B (33\%). Specificity was also highest for CLIA and LFIA kit B (both 100\%) followed by LFIA kit A (97\%). PPV and NPV were high for all assays with the exception of PPV for LFIA kit A (60\%) (Table 5).

Accuracy of serological tests for RT-PCR positive subjects $(n=75)$ revealed high sensitivity $(76 \%, 80$, and $69 \%$, respectively) for all serological tests. PPV were $100 \%$ for all tests.

For subjects with negative RT-PCR, sensitivity was 90\% for CLIA, $95 \%$ for LFIA kit A, and $100 \%$ for LFIA kit B. Specificity was $99 \%$ for CLIA and LFIA kit B, and $95 \%$ for LFIA kit A. PPV was $94 \%$ for CLIA, $70 \%$ for LFIA kit A, and $87 \%$ for LFIA kit B. NPV was high for all serological tests $(98.9 \%, 99.4 \%$, and $100 \%$ respectively).

\section{Discussion}

The aim of serological testing is to detect the presence of Abs against SARS-CoV-2 in the blood. These are specific proteins produced in response to infection and indicate that a person had contact with the virus. Antibody test results are important in detecting infection in patients with few or no symptoms, but it does not discriminate between ongoing and past infection. Several serological tests are available for surveillance and research purposes, but to date, their respective accuracy still remains scarce.

In our study, we analyzed 259 participants, including symptomatic patients undergoing COVID-19 testing based on clinical suspect and asymptomatic health care workers who had been exposed to infected persons. Three serological tests were used in parallel, one CLIA and two LFIA. The results show that the correlation among assay methods is optimal for IgG, but only fair for IgM. Furthermore, the sensitivity of CLIA is higher than LFIA in both patients and health care workers and the specificity is optimal for all diagnostic methods. CLIA is more sensitive and specific for the determination of both IgG and IgM, whilst the LFIA methods reported good sensitivity and specificity for IgG, but scarce sensitivity for the IgM determination. Further, sensitivity and the specificity of all 3 serological tests for subjects with RT-PCR positive and negative results were found to be adequate.

Currently, the diagnostic armamentarium available for the definition of SARS-CoV-2 infection status includes virus nucleic acid detection by RT-PCR, computer tomography (CT) imaging, and some hematological parameters. Furthermore, recent guideline [6] has also suggested the inclusion of other parameters which may help in the preliminary evaluation of patients' immune status and assessment of specific organ damage and oxygenation status, including leukocyte count, blood gas analysis, liver and kidney function markers, myocardial damage enzyme, myoglobin, erythrocyte sedimentation rate, $\mathrm{C}$-reactive protein, procalcitonin, lactate, D-dimer, coagulation damage, urine routine test, and inflammatory factors (interleukinIL-6, IL-10, TNF $\alpha$ ). 

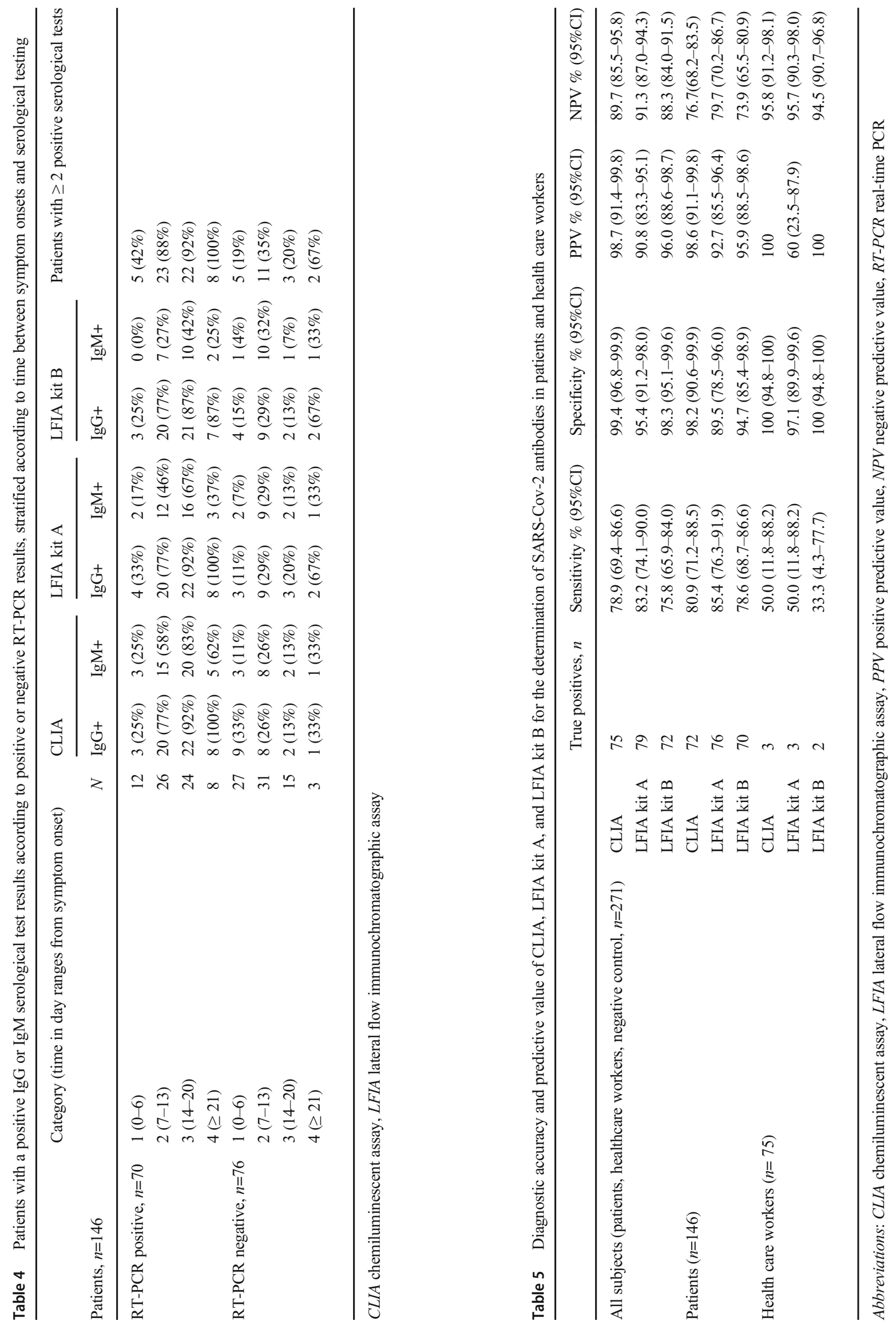
RT-PCR analysis on clinical specimens from patients with suspected COVID-19 is considered the confirmatory test for the diagnosis of SARS-CoV-2 infection by WHO and Centers of Disease Control and Prevention [5]. Although RT-PCR has high analytical specificity, it has some limitations. Firstly, RTPCR may generate false negative results [7], they must be elaborated in certified laboratories with expensive equipment, and thirdly, reported positive rates may vary for different swab specimens [8]. Diagnostic efficiency of RT-PCR depends on many additional factors, including sample type, stage of infection and distance from onset of symptoms [9], skill of personnel performing sample collection, and analytic performances of the PCR assays adopted [10].

Serological tests, which search for specific virus Abs, are available for use in clinical laboratories, but sensitivity and specificity outcomes are not uniform and the significance of their results is still under debate. Hsueh et al. [11] showed that IgM and IgA appear simultaneously and IgG could be detected as early as 4 days after symptom onset, in contrast to the well established antibody response profiles for acute viral infections. More recently, Li et al. [12] developed a rapid and simple point-of-care lateral flow immunoassay which can detect IgM and IgG antibodies against SARS-CoV-2 simultaneously in human blood within 15 min. Further, this test can detect patients at different infection stages, and the authors reported overall testing sensitivity of $88.7 \%$ and specificity of $90.6 \%$. However, a recent Italian study evaluated an emergency department patient population with LFIA and reported sensitivity of $18 \%$ and specificity of $92 \%$, with NPV of $26.2 \%$ and PPV of $87.5 \%$ [13]. Our current study reports an optimal sensitivity and specificity for serological tests in patients with suspected COVID-19 (>80\%). However, these results should be interpreted with caution as we included patients with either positive molecular tests or two positive, different serological tests in our definition of true positive, limiting the generalizability of our data.

IgM and IgG titers seem to be relatively low or undetectable in the initial days of infection and seem increase by day 5 [14]. Moreover, Zhao et al. [10] detected total Abs, IgM, and IgG against SARS-CoV-2 using immunoassays in 173 patients and reported that the presence of Abs occurred in $<40 \%$ of patients in the first 7 days of illness, but values increased to $100.0 \%, 94.3 \%$, and $79.8 \%$ for total $\mathrm{Abs}$, IgM, and $\mathrm{IgG}$ respectively since from day 15 after symptom onset. Likewise, Guo et al. [15] evaluated IgA, IgM, and IgG in 208 plasma samples using an ELISA based test. The median interval between symptom onset and IgM and IgA antibody detection was 5 days, while IgG was firstly detected on day 14 (IQR $10-18$ ), with positive rates of $85.4 \%, 92.7 \%$, and $77.9 \%$ respectively. Similarly, our results showed that $\operatorname{IgG}$ and $\operatorname{IgM}$ were present in serum of patients with RT-PCR positive after 6 days from symptom onset, and after 14 days, the sensibility and specificity were high $(>93 \%)$.
The development of IgG and IgM antibodies in COVID-19 patient blood is consistent with an acute viral infection; the IgG concentration begins to rise as IgM levels start to drop off. Usually, IgM antibodies can be detected as early as 3 days from the start of symptoms in many infections. IgM and IgG antibodies may be detected with immunoassay, such as an ELISA test, which requires a less stringent specimen quality than RT-PCR.

In conclusion, our study reports an adequate accuracy for serological tests for both patients and health care workers performing tests around 3 days from molecular test, allowing identifying potentially asymptomatic subjects in the first phase of the pandemic. However, our results suggest the IgG determination in patients infected by the SARS-CoV-2 virus may be useful to detect infection 6 days from symptom onset with adequate sensitivity and specificity. In fact, to understand the core element of the SARS-CoV-2 infection, a correct balance between sensitivity and specificity is essential not only to limit the frequency of false positive and false negative results but also to clarify the clinical sign and epidemiological evolution of the disease.

Supplementary Information The online version contains supplementary material available at https://doi.org/10.1007/s10096-021-04224-3.

Acknowledgements The authors would like to thank Johanna Chester for her critical revision and editing assistance.

Author contribution All the authors have accepted responsibility for the entire content of this submitted manuscript and approved submission. MS conceived and designed the study. MS, VP, TC, MM, TT, and GC entered and analyzed the data. All authors interpreted the data, prepared the full review, and contributed to its revision, interpretation of results, and approval.

\section{Declarations}

Ethical approval The study was conducted in accordance with the International Coordinating Council for Clinical Trials and the Helsinki Declaration and was approved by the Local Ethics Committee of our institution (No. 396/2020/OSS/AOUMO).

Conflict of interest The authors declare that they have no conflict of interest.

Guarantor VP.

\section{References}

1. Shanmugaraj B, Siriwattananon K, Wangkanont K, Phoolcharoen W (2020) Perspectives on monoclonal antibody therapy as potential therapeutic intervention for Coronavirus disease-19 (COVID-19). Asian Pac. J Allergy Immunol 38(1):10-18

2. Gallagher TM, Buchmeier MJ (2001) Coronavirus spike proteins in viral entry and pathogenesis. Virology 279(2):371-374 
3. Zhang J, Liu J, Li N, et al (2020) Serological detection of 2019$\mathrm{nCoV}$ respond to the epidemic: a useful complement to nucleic acid testing. medRxiv [Internet]. Available on :https://t.co/SrZF1qpwul

4. Bossuyt PM, Reitsma JB, Bruns DE et al (2015) STARD 2015: an updated list of essential items for reporting diagnostic accuracy studies. BMJ 351:h5527

5. Novel coronavirus (SARS-CoV-2) Discharge criteria for confirmed COVID-19 cases - when is it safe to discharge COVID-19 cases from the hospital or end home isolation?.TECHNICAL REPORT available on https://www.ecdc.europa.eu/en/publications-data/novelcoronavirus-sars-cov-2-discharge-criteria-confirmed-covid-19-cases

6. Jin YH, Cai L, Cheng ZS et al (2020) A rapid advice guideline for the diagnosis and treatment of 2019 novel coronavirus (2019nCoV) infected pneumonia (standard version). Mil Med Res 7(1):4

7. Yang Y, Yang M, Shen CG, et al (2020) Evaluating the accuracy of different respiratory specimens in the laboratory diagnosis and monitoring the viral shedding of 2019-nCoV infections. medRxiv published online Feb 17. DOI: https://doi.org/10.1101/2020.02.11.20021493

8. Chan JF, Yuan SF, Kok KH et al (2020) A familial cluster of pneumonia associated with the 2019 novel coronavirus indicating person-to-person transmission: a study of a family cluster. Lancet 395(10223):514-523

9. Zou LR, Ruan F, Huang MX et al (2020) SARS-CoV-2 viral load in upper respiratory specimens of infected patients. N Engl J Med 382(12):1177-1179
10. Zhao J, Yuan Q, Wang H, et al (2020) Antibody responses to SARS-CoV-2 in patients of novel coronavirus disease 2019. Clin Infect Dis ciaa344

11. Hsueh PR, Huang LM, Chen PJ et al (2004) Chronological evolution of $\operatorname{IgM}, \operatorname{IgA}, \operatorname{IgG}$ and neutralization antibody after infection with SARS-associated coronavirus. Clin Microbiol Infect 10(12): 1062-1066

12. Li Z, Yi Y, Luo X et al (2020) Development and Clinical Application of A Rapid IgM-IgG Combined Antibody Test for SARS-CoV-2 Infection Diagnosis. J Med Virol. https://doi.org/ 10.1002/jmv. 25727

13. Cassaniti I, Novazzi F, Giardina F et al (2020) Performance of VivaDiag COVID-19 IgM/IgG Rapid Test is inadequate for diagnosis of COVID-19 in acute patients referring to emergency room department. J Med Virol. https://doi.org/10.1002/jmv.25800

14. Zhang W, Du RH, Li B et al (2020) Molecular and serological investigation of 2019-nCoV infected patients: implication of multiple shedding routes. Emerg Microbes Infect 9(1):386-389

15. Guo L, Ren L, Yang S, et al (2020) Profiling Early Humoral Response to Diagnose Novel Coronavirus Disease (COVID-19). Clin Infect Dis ciaa310

Publisher's note Springer Nature remains neutral with regard to jurisdictional claims in published maps and institutional affiliations. 